CAROLINE CORNELIUS ${ }^{1}$

MARGARETE BOOS

\title{
Enhancing Mutual Understanding in Synchronous Computer-Mediated Communication by Training
}

\section{Trade-Offs in Judgmental Tasks}

Ineffective use of text-based synchronous computer-mediated communication $(C M C)$, that is, chats, may affect the quality of communicative exchange compared to effective use and to face-to-face (FtF) communication. Especially in groups making decisions in equivocal judgmental tasks, inappropriate use of the CMC medium often impairs performance. Users need high communication and media competencies to overcome the negative effects brought about by the technology. Without intervention, mutual understanding and satisfaction with the group process are reduced in computer-mediated decision groups. Training that helps participants adapt to the medium should provide them with the needed competencies. The authors found a complex pattern of process and outcome effects with the best performance scores in the FtF condition, performance scores in CMC with training approximating those of the FtF condition, and lowest performance scores in CMC without training.

Keywords: decision making; computer-mediated communication; mutual understanding; conversational coherence; training

Computer-mediated communication (CMC) systems offer some extraordinary advantages in permitting collaborative work over distances. But dispersed work teams must adapt to sociotechnical characteristics, for example, temporal delays in information and work flow and difficulties in maintaining shared context (Clark \& Brennan, 1993; Cramton, 2001; Walther, Boos, \& Jonas, 2002). In text-based synchronous CMC, participants can type and send messages simultaneously. However, the system does not deliver them in COMMUNICATION RESEARCH, Vol. 30 No. 2, April 2003 147-177 DOI: $10.1177 / 0093650202250874$

() 2003 Sage Publications 
COMMUNICATION RESEARCH • April 2003

the sequence intended by the participants. In addition, participants cannot see and hear one another and therefore are not able to regulate turn taking by nonverbal cues. These media features result in a chaotic flow of conversation in the CMC medium (McGrath, 1990), reduced transmission of interpersonal cues (Kiesler \& Sproull, 1992), and an enhanced need for and salience of situational social context cues (Spears \& Lea, 1992). Asynchronous computermediated groups and the systems that they use often utilize certain features that promote intermessage connectedness across a series of postings, for example, threaded subject lines and partial quoting of previous messages. These facilities are not common in synchronous systems.

Short-term groups in particular show less effort in adapting to the medium and their communication partners than groups anticipating further interaction (Walther, 1994). Thus, short-term computer-mediated groups communicating synchronously experience adaptation difficulties and reduced motivation in getting to know each other. This results in serious negative effects on within-group communication quality (McGrath \& Hollingshead, 1994). The group communication process is impaired by a lack of mutual understanding, which is accompanied by diminished satisfaction with the decision process and outcome (Straus, 1996).

The more a group task requires communicative exchange, the less satisfied short-term, computer-mediated, and synchronous groups are (Straus \& McGrath, 1994). Compared to idea generation and problem-solving tasks, decision making and other judgmental tasks require the highest coordination efforts of communicative contents. By definition, judgmental tasks have high equivocality, because they cannot be solved by finding a single correct or best answer (McGrath, 1984). Integration of divergent perspectives and agreement on a group decision presupposes mutual understanding (Dennis \& Valacich, 1999).

According to Dennis and Valacich (1999), features inherent to the media alone do not determine group effectiveness; media use and experience play a role as well. This study examines the antecedents and consequences of reduced mutual understanding in decision-making groups. Media comparison between conditions most extremely differing in mutual understanding and satisfaction, that is, face-to-face (FtF) groups versus synchronous computer-mediated groups of inexperienced users, may provide an approach to a better understanding of which communicative devices affect mutual understanding. To manipulate media use and experience, training aimed at supporting the adaptation to the sociotechnical characteristics of synchronous CMC is exerted and evaluated. We first discuss aspects generally required for creating mutual understanding and relate those requirements to the media features in CMC. Then we comment on the relevance of mutual 


\section{Cornelius, Boos • Enhancing Mutual Understanding}

understanding in decision-making groups in general and consider the consequences of reduced mutual understanding in CMC.

\section{General Requirements of Mutual Understanding}

Mutual understanding presupposes a common ground consisting of shared information, mutual knowledge, mutual beliefs, and mutual assumptions (Clark \& Carlson, 1982). In the process of grounding, conversation participants seek positive evidence of understanding by the following sources of feedback: acknowledgments by back-channel responses, continued attention, and the initiation of the relevant next turn (Clark \& Brennan, 1993). Relevant next turns can be produced in every kind of media, whereas backchannel responses and signaling continued attention by eye gaze require the copresence of $\mathrm{FtF}$ communication.

What kind of turns are relevant to the preceding turn? Sperber and Wilson (1986) argued that conversation participants feel free to choose the relevant context for a turn as well as its interpretation on a moment-to-moment basis in order to encode or decode messages appropriately. Speakers establish mutual understanding by framing and bridging their utterances, constructing as many feedback loops as necessary in order to coordinate meaning and to agree about the shared context (Clark \& Wilkes-Gibbs, 1986). Senders encode their messages by choosing the frame that will facilitate recipients' choice of the right bridge to mutual understanding.

Conversation is inherently dyadic - a continuing dialogue between two people. This is the case not only in dyads but in larger groups, too. Two group members hold the floor in common for relatively extended periods of time, taking turns as speaker and addressee, with other group members being allocated to listener roles (Reid \& Ng, 2000; Zajonc, 1960). Such dyadic exchanges make up $61 \%$ of turns in four-person decision-making groups (Parker, 1988) and $49 \%$ of turns in six-person decision-making groups (Stasser \& Taylor, 1991). Sometimes this dyadic exchange is broken by a third group member, and the floor is regained from the initial dyad for floor sharing by other dyads in the group. But nondyadic exchange-floor turns not dyadically shared-is observed rarely in FtF groups (22\% in four-person groups; see Stasser \& Taylor, 1991; 9\% in six-person groups; see Parker, 1988).

Dyadic exchange involves dynamic changes of speaker and addressee roles. The current addressee sends feedback that is not restricted to back channels but includes all communicative devices dependent on a preceding speech act: answering questions, showing agreement or disagreement, acknowledging, and referring to the speaker. 
COMMUNICATION RESEARCH • April 2003

In dyadic exchanges competent communicators use their knowledge about the encompassing contexts to encode and decode messages appropriately (Planalp \& Tracy, 1980). They relate their messages to previous topics when introducing new information (Clark \& Haviland, 1982). Hence, they take into account the frame of reference of their communication partners, enabling them to infer the intended meaning by choosing the right bridge between message and context (Clark \& Wilkes-Gibbs, 1986). As a result, the immediate verbal context is the most relevant frame of reference for a message. If a given message cannot be interpreted in this context, the receiver of the message will search for interpersonal context cues to infer meaning. When the communication partners are unfamiliar with one another or do not succeed in finding an interpersonal frame or bridge due to a lack of interpersonal context cues, they will choose a broader social context to encode or decode the message. We therefore might expect unfamiliar short-term groups communicating in a visually anonymous medium without previous chat experience to share only a minimum of common ground and to have only poor possibilities of grounding.

The immediate verbal context serves as a frame of reference if it is coherent. Conversational coherence can be defined as the joint product of participants' abilities and motivation to refer to each other and to develop topics. If participants are not able or motivated to fulfill the basic requirements of framing and bridging, conversational coherence and mutual understanding cannot be established and communication will break down. Conversational coherence is a prerequisite of mutual understanding. Mutuality can never be built up by the communication and media competence of a single group member. Each group member has to contribute and process information in a way that leads to a shared mental model of the conversation (Reichman, 1978). Conversational coherence is supposed to serve two basic functions of conversation management: the management of conversation flow and the management of interpersonal relationships. Noncoherent conversation management would lead to disruptive, fragmented topical flow and at the same time should be accompanied by low interest and politeness and friendliness toward other group members and their topics (Cappella, 1994; Ng \& Bradac, 1993; Tracy, 1985). Unfortunately, these assumptions have never been validated empirically before.

\section{Requirements of Mutual Understanding in CMC}

Miscommunication can also take place if participants do not consider the constraints of the communication medium that provides the physical context for 


\section{Cornelius, Boos • Enhancing Mutual Understanding}

the communication. This can occur, for example, when participants in CMC produce messages as if they were working in a $\mathrm{FtF}$ communication situation. To give an example, maintaining topics is relatively effortless in $\mathrm{FtF}$ communication. However, in synchronous CMC all participants can generate and send messages simultaneously. Therefore, clarity about the topic and sequence is more effortful. The positive evidence of understanding by initiating a relevant next turn is often missing. Moreover, the nonverbal feedback that is available in FtF can only be compensated for in CMC by costly verbal feedback. Explicit references to topics and communication partners are required in order to make each message relevant to the verbal context. Furthermore, the dyadic exchange in groups, which produces dyadically shared floor taking, is impossible without directly addressing the other group members by name in CMC. When users do not adapt their communicative behavior to the medium, the immediate verbal context is eliminated and lack of coherence follows (Herring, 1999).

In chats, the simultaneous exchange of messages imposes a cognitive load on participants, which results in less attention to and less orientation toward the contributions of other group members. In the chaotic conversational environment of CMC, group members are so occupied typing and reading messages and developing their own ideas that they have few cognitive resources left to devote to attending to other group members (Straus, 1996). References to the contributions of other group members, deliberate evaluation of the arguments of others, answering questions, and any other kind of feedback may be affected by such attention blocking, and this will further accentuate the lack of coherence and mutual understanding in CMC.

Hypothesis 1a: In synchronous CMC groups, coherence is lower than in FtF communication.

Additionally, the process of grounding will be disturbed by the unfamiliarity of short-term group members and the lack of media-adapted conversation strategies of inexperienced users:

Hypothesis 1b: Groups with low chat experience and little familiarity between group members show a lower degree of coherence than groups who are highly familiar with each other and the media.

The grounding process is organized in speaker-addressed dyads within groups. If group discussion is less dyadically organized, common ground cannot be established by feedback loops: 


\section{COMMUNICATION RESEARCH • April 2003}

Hypothesis 1c: In synchronous CMC groups less speech is organized in dyadic floor sharing than in $\mathrm{FtF}$ groups.

Communicators rarely perceive incoherence as a result of constraints of the communication media. When adaptation failures occur, group members are prone to make dispositional attributional judgments about distant partners rather than consider their own adjustment difficulties (Cramton, 2001; Walther et al., 2002). Because of the fundamental attribution error, they blame results such as incoherence on low ability and motivation on the part of their communication partners to show interest and friendliness (Bradac, 1988). At the same time, the need for coherence can evoke a coherence bias, so that participants detect interconnectedness and meaningful relationships even if no coherence was intended at all (Hellmann, 1995; Schwarz, 2000; Werner \& Latané, 1976). Thus, the fundamental attribution error leads to interpersonal misunderstandings, whereas the coherence bias leads to miscommunication of content. Sufficient coherence may prevent group members from fundamental attribution errors and misunderstandings:

Hypothesis 2: The degree of coherence will be positively related to the level of mutual understanding and interpersonal attraction.

\section{Mutual Understanding as a Requirement of Effective Decision Making}

The dynamic interdependence of speakers, addressees, and other listeners in creating meaning is increased if they interact in a group with the task requirement being to come to a mutually agreed-upon solution. This kind of convergence in meaning is necessary in decision-making tasks.

The final aim of decision-making groups is coming to a consensus. In cohesive groups consensus may be a result of conformity and other in-group pressures rather than the result of deliberate integration of information and meaning (Janis, 1972; but see Hogg, 1992). Small groups with a common history direct their communication and decision processes by already established norms, roles, and routines (McGrath, 1984). But even in short-term groups with zero history, the social influence of group membership on decision making may result in group polarization of attitudes toward an extreme norm of the social group (Turner, 1991). Thus, the plain fact of coming to consensus is not necessarily evidence of the quality of decision making. A crucial criterion for the quality of performance is the quality of the decision-making process leading to consensus. There often is no other clear criterion against which the quality of the decision may be assessed, especially in experi- 


\section{Cornelius, Boos • Enhancing Mutual Understanding}

mentally created short-term groups with equivocal decision-making tasks (Brown, 2000). To obtain well-deliberated and well-integrated decisions, it is not enough to prevent group members from using group norms as their main basis for choice, because this may result in divergence instead of convergence. On one hand, divergence fosters diversity of opinions, but on the other hand, it complicates reaching a consensus. High quality communicative exchange and mutual understanding are needed to be able to manage the diversity of opinions and the equivocality of judgmental tasks.

The higher the coordination requirements of a task, the more critical the effect of communication on task performance will be. Coordination in decision-making groups requires communicative exchange, communicative competencies, and the appropriate use of the communication media for the task being carried out. This is not only valid for the availability of opportunities to communicate (Reimer, Neuser, \& Schmitt, 1997) and for the quantity of exchanged information (Boos \& Sassenberg, 2001) but also for the quality of communication (Tschan, 1995). Whenever a group has to establish a shared model of the task and has to integrate divergent perspectives in a joint problem solution, mutual understanding is crucial for task completion. Compared to idea generation and problem-solving tasks, judgmental tasks require the

highest amount of communicative coordination, because task completion requires extensive feedback, that is, agreement or disagreement and task focus (McGrath, 1984; Straus, 1999).

Moreover, in coming to consensus, group members are highly interdependent. They have to develop a shared cognitive representation of the problem; a common orientation toward a goal; evaluations of facts, opinions, and decision proposals; and shared criteria for evaluation (Boos \& Sassenberg, 2001). Effective coordination of these task contents does not always imply that the group comes to consensus but to a shared perspective of these contents. Not achieving consensus can be an appropriate group decision, too.

If members detect and decide that they do not share the same problem definitions, goals, and criteria for evaluation, they have established a metaconsensus. Consequently, the criterion for task completion is not reaching consensus but satisfaction with the decision process in terms of effective coordination. These include being satisfied with the problem definition, with the integration of perspectives, and with the result of the discussion, particularly if the group did not come to the demanded consensus.

Conversational coherence fosters a shared cognitive representation of the task and its requirements, thus increasing task focus and feedback toward the uttered opinions and decision proposals of the other group members, providing the group with the required communicative resources for task com- 
COMMUNICATION RESEARCH • April 2003

pletion. In synchronous CMC, low coherence and mutual understanding may be insufficient to fulfill these prerequisites of decision making:

Hypothesis 3: Compared to FtF groups, CMC decision-making groups without training are lower in the amount of task focus, the amount of feedback, and also in their satisfaction with the group process.

Coherence and mutual understanding are necessary and sufficient predictors for satisfaction with the decision process, whereas attaining consensus may predict satisfaction but is neither necessary nor sufficient for it.

But can we trust satisfaction ratings if the group is highly cohesive? Irrespective of the effective coordination of task requirements, cohesive groups tend to be highly satisfied with their decision process. If group members confuse interpersonal attraction with mutual understanding, we are more likely to find coherence biases that result in inappropriate evaluations (such as, "I didn't understand a word, but I agree"). Interpersonal attraction may be higher in consensus groups than in nonconsensus groups (Lott \& Lott, 1965), suggesting an alternative route to satisfaction with group processes in consensus groups compared to nonconsensus groups. If group members feel attracted because of their high commitment to the group, they will overrate the quality of their group process and outcome. This relationship between interpersonal attraction and perceived mutual understanding occurs irrespective of coherence and is assumed to influence the perception of satisfaction in consensual groups.

In FtF groups, interpersonal attraction and mutual understanding may work toward consensus either independently or in combination. In CMC without media-adapted conversation management, interpersonal attraction and mutual understanding may be perceived but have no measurable counterpart in communicative exchange (such as coherence, dyadic organization of talk, feedback, and task focus). This kind of interpersonal attraction may foster the attainment of consensus:

Hypothesis 4: In consensus groups, interpersonal attraction will be positively related to satisfaction with the decision process.

In nonconsensus groups, interpersonal attraction may not influence satisfaction. But meeting task requirements by well-structured coordination process may compensate for the failure to reach consensus:

Hypothesis 5: In nonconsensus-groups, interpersonal attraction will be positively related to satisfaction with the decision process. 


\section{Cornelius, Boos • Enhancing Mutual Understanding}

Several earlier studies showed that short-term CMC groups have more difficulties in reaching consensus if they experience time pressure (for an overview see Walther, Anderson, \& Park, 1994). Thus, we expect more nonconsensus groups in the CMC condition than in the FtF condition:

Hypothesis 6: When groups are working within tight time restrictions, $\mathrm{FtF}$ groups come to consensus more often than do CMC groups.

In sum, we expect less consensus and less coherence in synchronous inexperienced computer-mediated decision-making groups. Therefore, in this condition, satisfied groups will rarely be observed.

\section{Media-Adapted Communication in Chats}

In small FtF groups, participants are physically copresent, thus automatically transmitting a substantial number of personal cues. They engage constantly in feedback activities and talk to each other in a clear sequence of speakers (McGrath, 1990). Therefore, FtF appears to be the best fitting medium to permit coherence, reduce equivocality, and foster mutual understanding and convergence toward consensus. Synchronous computer-mediated short-term groups seem to be located at the negative end of the task-media-fit dimension: Feedback can only be transmitted by costly verbal cues, back-channel behaviors are filtered out, and participants often engage in parallel conversations (Herring, 1999).

However, media use is variable. CMC may be even more personal than FtF communication if participants expect future interaction, are familiar with each other, and are motivated to further develop interpersonal relationships (Walther, 1996). Earlier approaches based solely on media features neglect the adaptability of users to the medium. Training of media-adapted conversation management strategies could convince participants that the benefits of coherence are higher than the costs of not establishing a shared communicative environment.

Such training should include several features. First, explicit references to the topics of other group members by directly addressing and repeating key words can enhance coherence. Second, asking questions and giving answers should establish a sequential structure of communication, even if the sequence has been disrupted due to simultaneous generation of messages. Both topic and source reference, and the use of questions and answers, can increase coherence and can facilitate reconstruction of the conversation (Kent, Davis, \& Shapiro, 1978; Schegloff, 1990). Direct addressing of communication partners' names may reestablish the dyadic structure of conver- 
COMMUNICATION RESEARCH • April 2003

sation. And last but not least, a preceding training of the group fosters familiarity. Such trained CMC groups will be involved in grounding processes and orient their talk to their communication partners and topics. So hypothesis 1 has to be extended.

Hypothesis 1d: A combined training of reference and request strategies enhances coherence compared to CMC groups without training.

Because no previous research on this issue has been done, we need to explore whether a combined training of both strategies or just a training of one of the strategies is sufficient to enhance coherence and mutual understanding in CMC. Because establishing coherence is assumed to be not only a skill but also motivated by affiliation, the trained group members should be familiar with each other and expect future interaction outside the laboratory.

Enhancing coherence and mutual understanding in familiar CMC groups should enable participants to focus on the task and to coordinate task contents. Consequently, the effect of poor media adaptation on coordination should be reduced, and satisfaction with the coordination provided by the group process should be enhanced. However, we do not expect trained CMC groups to come to consensus more often than CMC groups without training. Creating coherence and mutual understanding in chats is time costly and will only enhance the quality of the decision process but not the consensus frequency.

More central to our approach is the fact that poor media use offers the opportunity to study the effects of noncoherence and of a lack of mutual understanding on decision making without manipulating social context factors, such as in-group or out-group identity (Oehlschlegel \& Piontkowski, 1997; Sassenberg \& Postmes, 2000). If training in strategies aimed at increasing coherence can compensate for low mutual understanding in CMC, this would not only suggest explanations for impaired group processes in CMC but would also provide a basis for the development of effective intervention methods.

\section{Method}

Sample

Participants were 240 students attending a German university. They were randomly assigned to 80 three-person groups. Forty groups were in the FtF condition, 10 groups were in a CMC condition without training. Ten groups 


\section{Cornelius, Boos • Enhancing Mutual Understanding}

were in each of the three conditions receiving forms of communication training. Data from only 38 of the FtF groups could be included in the sample because the communications of one group could not be coded due to equipment failures and one group (the only all-male group) contributed extreme values to the data. Only 37 of the CMC groups could be included in the sample due to data loss because of server breakdowns. Twenty-nine percent of the FtF groups were all female, $71 \%$ had both male and female members; $65 \%$ of the CMC groups had both male and female members, $13.5 \%$ were all female, and $21.5 \%$ were all male. We wanted members to be familiar with one another so that they would expect future interaction and in order to enhance grounding processes and motivation to be coherent. Unfortunately, $19 \%$ of the participants met for the first time in the laboratory. Group type was coded for the degree of familiarity: identifiable (the members know each other), familiar (the members know each other for a longer period), and friends. ${ }^{2}$ There were 27 groups of friends, 28 familiar groups, and 19 identifiable groups in the sample. In 4 CMC groups no member had previous chat experience, in 15 CMC groups a single member had previous chat experience, in $15 \mathrm{CMC}$ groups two members had chatted before, and in 3 CMC groups all members had chatted before. Because gender composition, familiarity of group members, and chat experience could not be controlled, they were coded as dummy variables and entered in each analysis. Eighty-seven percent of the sample reported poor typing abilities. Most surprisingly, $40 \%$ of this student population reported having never or seldom used a personal computer before.

Task

Groups worked on a career-choice dilemma similar to the judgmental tasks used by Kogan and Wallach (1964) in the group polarization paradigm. The key question they needed to address was, "How high does the chance of later employment have to be before you would advise a fellow student to join a very desirable trainee program prior to finishing the undergraduate thesis?" Each participant was first asked to mark her or his individual judgment on a scale ranging from $10 \%$ to $100 \%$ and then received instructions to discuss the issue with the other group members and to come to a consensus on how high the chance of employment needed to be in order to advise the fellow student to join the program. After group discussion, individual judgments were again obtained. In contrast to intellective tasks, the outcome of this judgmental task is "subject to multiple and conflicting viewpoints that cannot be readily resolved through the presentation of factual information" (Straus, 1999, p. 170). 
COMMUNICATION RESEARCH • April 2003

\section{Procedure and Communication Training}

At their arrival, participants of both media conditions were asked into a room with a round table where they were to get familiar with each other, if necessary. Before group discussion, each participant in the CMC condition completed a short, online questionnaire in a separate room, received the group task and instructions at the computer terminal, marked the individual judgment on a scale from $10 \%$ to $100 \%$, and then entered a chat facility (Netmeeting 2.0 by Microsoft). In the FtF condition, participants were seated around a table and were videotaped. After group discussion all members completed a longer online questionnaire. Finally, all group members met again FtF, were rewarded with a lottery ticket, and debriefed.

Both media conditions had their discussions within a given time frame. The experimenter interrupted the FtF groups after 10 minutes. CMC groups received 4 times more discussion time in order to diminish the effect of the typing effort (Walther, 1996). After 40 minutes discussion time, CMC groups were interrupted as well.

Groups in the experimental training conditions received a 15-minute oral presentation accompanied by transparencies illustrating competent conversation management in CMC. Ten groups participated in the control condition (CMC without training) and were given a "placebo" communication training. They were told that most of the difficulties in CMC arise from the fact that people do not know each other and are not familiar with the electronic communication technology. They were instructed to take 15 minutes to talk to each other directly and then to get used to the technology by chatting online about the pros and cons of the medium for another 20 minutes before finally starting to work on the group task.

Thirty groups were randomly assigned to three training conditions. Each kind of training started with an illustrative example of miscommunication that can result from the simultaneous generation of messages in the CMC medium. In the following example, the difficulty of keeping focused on the topics and on other members while participating in electronic conversation are described:

(Example 1: Translated contents of the first transparency of the reference training)

Johanna: Of course, under these circumstances feeling of togetherness is low. Mathias: If informal communication was fine, people did a good job, often had fun. Sabine: I have to agree, too much energy wasted . . .

Johanna: This might have led people to not being dedicated to the company, irrespective of their identification with the product... 


\section{Cornelius, Boos • Enhancing Mutual Understanding}

Sabine: What do you think of our discussion style?

Johanna: Sorry, my last two sentences belonged together.

Mathias: This needs getting used to.

Johanna: It's a little bit of a chaos. You type something and suddenly it does not fit any longer/it doesn't match the previous statement. You can't really refer to anything.

Sabine: We did not even introduce ourselves.

Mathias: This kind of communication will train our flair as detectives! Best regards, Sherlock Holmes.

After this problem sensitization, the proposed solution strategies were offered, illustrated with better adapted computer-mediated dialogues on four further slides: In the condition CMC with reference training, participants learned to address the other individual members explicitly and to indicate the topic of the message to which they were responding. In the condition CMC with request training, they were taught to structure conversation in terms of requests and responses. In the condition $\mathrm{CMC}$ with combined training, participants learned both strategies. After the presentation participants were accompanied to different rooms, were introduced to and instructed in Netmeeting, and then were asked to rehearse the learned strategies in a 20minute online discussion about the pros and cons of the medium.

Before all CMC groups started the computer conference they had to complete a questionnaire measuring familiarity with the technology and with the other group members and also assessing demographic data. After the online discussion about the pros and cons of the medium, groups received the task instructions, responded individually to the decision scale, and received the instruction to come to a consensus in the decision dilemma of the fellow student. After 40 minutes participants had to finish the computer conference, regardless of whether they had reached a consensus. The computer session was closed with the completion of the questionnaire measuring mutual understanding, satisfaction with the group process and outcome, and interpersonal attraction.

\section{Dependent Variables}

Questionnaire Scales

The 6-point scale Mutual Understanding included the following five items: "I was able to understand the point of view of the others," "I could make myself heard," "The others showed interest in my opinions," "I could follow the flow of conversation," and "The others referred to me." All items loaded higher than .60 on the first factor of a varimax-rotated principal components analysis with Kaiser normalization criterion equal to 1 . The first factor explained $32 \%$ 


\section{COMMUNICATION RESEARCH • April 2003}

of the variance in the sample. The scale had good internal consistency (Cronbach's $\alpha=.84$ in FtF and $\alpha=.85$ in CMC groups).

The 6-point scale Satisfaction With the Group Process was composed of the following items: "I could extend my perspective by the ideas of the others," "We defined the problems adequately," "In my opinion, the discussion was effective," "We worked together in a cooperative manner," "I am satisfied with our result (even if no decision was made)." All items loaded higher than .60 on the second factor, explaining $15 \%$ of variance. The scale showed a good reliability in both media conditions ( $\alpha=.81$ in FtF, $\alpha=.74$ in CMC).

The 6-point scale Interpersonal Attraction consisted of the items "We had a pleasant discussion," "I would like to work again with my partners," and "My partners were likable." The items loaded with .60 or more on the third factor, explaining $8 \%$ of variance. Reliability was sufficient $(\alpha=.72 \mathrm{in} \mathrm{FtF,} \alpha=$ .77 in CMC).

The other items originally included in the questionnaire loaded less than .50 on the remaining factors. The remaining factors explained less than $5 \%$ of the variance and were not used for further statistical analysis.

\section{The Coding of Coherence and Other Process Variables}

The data source for coding were 38 transcripts of videotaped FtF discussions and 37 printed protocols of computer conferences. The following list gives an outline of the coding systems (the single categories will be defined in detail below):

- Topic management categories (Crow, 1983);

- Explicit reference categories (as included in the training);

- Requests and compliances with requests (as included in the training);

- Statements and feedback categories (Condon \& Cech, 1996);

- Task orientation, steering acts, socioemotional orientation (Bales, 1950) and medium-related content;

- Direct addressing (as included in the training of reference strategies).

The coding unit for all category systems was a given turn defined by the turn taking of the speakers. In CMC, a turn was identical with a sent message. Sometimes a turn was composed of several topics. In these rare cases, which occurred only one to three times in a discussion, we decided to break down a turn into as many subturns as topics discussed.

Topic management categories were coded with the procedure defined by Crow (1983): (a) coherent devices such as "maintaining current topic," "topic shading," "renewal of group topic," and "renewal of the topic given by a 


\section{Cornelius, Boos • Enhancing Mutual Understanding}

Table 1

Interrater Reliability Coefficients Between Three Coders ( $\mathrm{n}=347$ coding units)

\begin{tabular}{lcl}
\hline & \% of Agreement & $\kappa$ \\
\hline Coherence & 71 & $.61^{* * *}$ \\
Explicit reference & 48 & $.53^{* * *}$ \\
Requests & 57 & $.69^{* * *}$ \\
Compliances with requests & 33 & $.29^{* * *}$ \\
Feedback & 71 & $.58^{* * *}$ \\
Task orientation $^{\mathrm{a}}$ & 82 & $.75^{* * *}$ \\
Direct addressing $^{\mathrm{a}}$ & 66 & $.87^{* * *}$ \\
\hline
\end{tabular}

a. In the face-to-face transcript there was no incident of direct addressing.

*** $p<.001$.

communication partner"; and (b) noncoherent topic shifts such as "inserts," "initiation of a new topic," and "insisting on own topic." The coefficient of coherence (CC) is estimated by calculating the difference between the sum of messages coded in the coherent topic management categories and the sum of messages coded in the noncoherent topic management categories divided by the sum of messages in the coherent plus noncoherent categories. This procedure permits comparisons between groups that work for unequal periods of time, or have unequal rates of interaction.

Interrater reliability was estimated by percentage of agreement and Cohen's kappa coefficient ( $\kappa$; see Table 1). The kappa coefficient takes into account the level of coding agreement that would occur by chance (Cohen, 1960). $\kappa=0$ indicates just chance agreement, $\kappa>0$ indicates more than chance agreement, and $\kappa=1$ indicates perfect agreement. Kappa coefficients should differ from 0 at the .01 level of significance or better (Fleiss, 1981). However, Bakeman and Gottman (1986, p. 82) claimed that interrater agreement should not just be better than chance but "good." There exists no statistical rationale to estimate the goodness of kappa coefficients but only informal rules of thumb. According to Fleiss (1981, p. 218), kappas greater than .80 are interpreted as excellent, kappas between .60 and .75 as good, and kappas of .40 to .60 as fair. Bakeman and Gottman already regard kappas less than .70 with some concern. However, every rigid assessment scheme of the range of kappa coefficients should be handled with care, because the evaluation is dependent on the experiences of the individual researchers and their research subject.

All coding was done by the first author after several months of training and after reaching an interrater reliability of $\kappa=.84$ with the second author. A student research assistant was trained in the coding method, and interrater agreement between the first author (who coded the entire data) and the second author and the student research assistant (who each coded 
COMMUNICATION RESEARCH • April 2003

half of the data) was calculated. The three coders analyzed 347 coding units from the four forms of group discussions.

Topic management categories yielded an agreement of $\kappa=.46$, which was interpreted as too low for further statistical analysis. However, calculation of the $\mathrm{CC}$ was based solely on the sums of coherent and noncoherent topic management categories, without any differentiation among single topic management categories being made. Interrater agreement of coherent and noncoherent turns yielded an interrater agreement of $\kappa=.61$, which Fleiss (1981) would regard as good (see Table 1).

Explicit topical and source references were coded in order to obtain a score for the efficiency of the reference training. The trained devices, explicit agreement, explicit disagreement, topical reference, and summaries made were coded and yielded an agreement of $\kappa=.53$, which Fleiss considers fair reliability. A suboptimal reliability reduces the power of finding effects, that is, raises the likelihood of Type II error.

A further score for the efficiency of the training was requests and compliances with requests. The feedback score is a frequency count of agreements and disagreements, a crucial process measure in judgmental tasks (Straus, 1999). Requests, compliances with requests, and feedback were coded with a strategy similar to the coding system of functional categories in decision making used by Condon and Cech (1996). The authors distinguished between move functions such as requests for information and action, response functions such as agreement, disagreement, and responses to requests, and other functions such as meta-language and personal information. Condon and Cech reported a high reliability (80\%-100\%) for frequently used categories but an insufficient reliability for infrequently used categories. In an attempt to increase the reliability, we used separate coding steps for requests, responses, feedback, and the context of the move/response category.

Intercoder reliability for request was good, $\kappa=.69$, but the reliability for coding compliance was unacceptable, $\kappa=.29$. Because the coders often confused compliances with requests and feedback and subsequent attempts to differentiate them proved unsuccessful, we chose to drop compliances from the analysis. The intercoder reliability for feedback was fair (Fleiss, 1981), achieving a kappa coefficient of .58.

The percentage of task-oriented contributions was used as an index of task focus of the group. The context of every turn was specified using four broad categories: task orientation, steering, socioemotional, and medium-related content (see Bales, 1950, for a similar procedure). The kappa of .75 allows further statistical analysis. 


\section{Cornelius, Boos • Enhancing Mutual Understanding}

In CMC, the dyadic organization of talk is measured by the degree of direct addressing. Direct addressing using the partner's name almost never occurs in $\mathrm{FtF}$ because participants direct their speech by body posture, eye gaze, and so forth. Direct addressing in FtF is therefore operationalized by these nonverbal cues and was coded in the "To whom" column of the transcripts. For the transcripts, no coding reliability was calculated (all transcripts were done by the first author). In CMC, direct addressing is operationalized as an explicit verbal source reference and easy to comprehend for participants as well as for conversation coders, a fact reflected in the high interrater reliability of $\kappa=.87$.

\section{Results}

\section{Effectiveness of Forms of Training}

First, we tested the prediction of reduced coherence in CMC compared to FtF (Hypothesis 1a). Second, we compared the efficacy of the combined training in enhancing coherence in CMC and compared the efficacy of both strategies (see Hypothesis 1d). The training of explicit reference should increase the rate of references, the training of requests should increase the rate of requests, and the combined training should increase both (see Table 2).

Coherence was lower in CMC without training compared to FtF. Every kind of training (combined, reference, and request) was equally effective in enhancing coherence compared to no training. Chat experience and gender composition had no effect on coherence (all $F$ scores $<1.0$ ). However, low familiarity affected coherence negatively, $F(1,68)=5.3, p<.05$ (see Hypothesis 1b). Familiar groups and groups of friends did not differ significantly in coherence.

Which strategy was more appropriate to create coherence in CMC? Compared to groups without training, groups who received the combined training chose to use the reference strategy more often than the request strategy. They therefore were equally coherent to groups who only received the reference training. Compared to FtF, question asking was significantly greater in the untrained CMC condition. Even in groups that received the request training, fewer requests occurred than in the untrained condition, but training in general reduced posing questions. Consequently, it seems more promising to enhance coherence by explicit reference than by requests.

Whereas chat experience and gender composition of the group had no effect on the amount of references (all $F$ scores $<1.0$ ), familiarity did, $F(1,75)=4.7, p<.05$, following the same pattern as the effect of familiarity on coherence. 
Table 2

A Means, Standard Deviations, Contrast Estimates, and F Statistics of ANOVAs for Effectiveness of Training Coherent Strategies in ComputerMediated Groups with Chat Experience, Familiarity, and Gender Composition of the Group as Covariates

\begin{tabular}{|c|c|c|c|c|c|c|}
\hline Group Condition & Process Variable & $M(n=75)$ & $S D$ & Contrast Estimate $^{a}$ & $F d f=4,68$ & $p$ \\
\hline & Coefficient of coherence & & & & & \\
\hline CMC no training & & 0.29 & 0.18 & & & \\
\hline CMC combined training & & 0.58 & 0.02 & $0.28 * * *$ & & \\
\hline $\mathrm{CMC}$ reference training & & 0.52 & 0.18 & $0.22 * * *$ & & \\
\hline CMC request training & & 0.52 & 0.15 & $0.24 * * *$ & & \\
\hline \multirow[t]{2}{*}{ Face to face } & & 0.68 & 0.10 & $0.38 * * *$ & 19.5 & .000 \\
\hline & Explicit references & & & & & \\
\hline CMC no training & & 0.04 & 0.04 & & & \\
\hline CMC combined training & & 0.26 & 0.08 & $0.21 * * *$ & & \\
\hline $\mathrm{CMC}$ reference training & & 0.24 & 0.08 & $0.19 * * *$ & & \\
\hline $\mathrm{CMC}$ request training & & 0.15 & 0.07 & $0.11^{*}$ & & \\
\hline \multirow[t]{2}{*}{ Face to face } & & 0.21 & 0.08 & $0.16^{* * * *}$ & 11.5 & .000 \\
\hline & Requests & & & & & \\
\hline CMC no training & & 0.28 & 0.05 & & & \\
\hline CMC combined training & & 0.23 & 0.04 & -0.04 & & \\
\hline $\mathrm{CMC}$ reference training & & 0.22 & 0.05 & $-0.05^{*}$ & & \\
\hline $\mathrm{CMC}$ request training & & 0.26 & 0.05 & -0.01 & & \\
\hline \multirow[t]{2}{*}{ Face to face } & & 0.13 & 0.07 & $-0.15^{* * *}$ & 33.43 & .000 \\
\hline & Direct addressing & & & & & \\
\hline CMC no training & & 0.05 & 0.01 & & & \\
\hline CMC combined training & & 0.26 & 0.10 & $0.21 * * *$ & & \\
\hline $\mathrm{CMC}$ reference training & & 0.34 & 0.09 & $0.27 * * *$ & & \\
\hline $\mathrm{CMC}$ request training & & 0.17 & 0.14 & $0.12 * *$ & & \\
\hline Face to face & & 0.72 & 0.08 & $0.66 * * *$ & 169.86 & .000 \\
\hline
\end{tabular}

Note: $\mathrm{CMC}=$ computer-mediated communication.

a. Contrast estimates were calculated by simple contrast analysis with the condition $\mathrm{CMC}$ without training as reference category. $* p<.05 . * * p<.01 . * * * p<.001$ 


\section{Cornelius, Boos • Enhancing Mutual Understanding}

Chat experience, gender composition, and familiarity of the group had no effect on the amount of requests (all $F$ scores $<1.0$ ).

Hypothesis 1c was supported. Dyadic exchanges operationalized as "direct addressing," using names in CMC and with eye gaze direction in FtF, was $72 \%$ in FtF and therefore higher than in Stasser and Taylor's six-person groups and Parker's four-person groups, perhaps due to the smaller group size in our sample. In CMC without training, only $5 \%$ of the talk is organized in dyadic shared-floor turns, leading to a large contrast between $\mathrm{FtF}$ and CMC without training. Although non-shared floor is standard in CMC, our results indicate that $\mathrm{CMC}$ groups with the combined training and groups with reference training have significantly higher levels of dyadic exchange than CMC groups without training. Groups with explicit reference training organize $34 \%$ of their talk in dyadic shared turns. The request training had a comparably smaller, less significant impact when compared to groups without training.

Chat experience and gender composition of the group had no effect on direct addressing (all $F$ scores < 1.0). Friends and identifiable groups addressed their messages more often to the whole group than to singledialogue partners, whereas familiar groups used direct addressing more often, $F(1,74)=5.3, p<.05$.

\section{Effects of Coherence on Perceived Mutual Understanding}

In Hypothesis 2 we stated that coherence predicts mutual understanding and interpersonal attraction. When all 75 groups and all sources of variance were included in the first step of a multiple regression analysis, measured coherence predicted perceived mutual understanding significantly, $\beta=.35$, $F(1,73)=10.0, p=.002$, adjusted $R^{2}=.10$, thus supporting the first part of Hypothesis 2. The second part of Hypothesis 2 is not supported: Coherence did not predict interpersonal attraction. The nonexisting relationship may be interpreted in terms of insufficient interrater reliability of the coherence index. However, interpersonal attraction and mutual understanding were significantly correlated: $\beta=.51, F(1,73)=25.6, p=.000$, adjusted $R^{2}=.25$.

Before we could test the effects of coherence, mutual understanding, and interpersonal attraction on satisfaction with the group process (Hypotheses 4 and 5), we had to consider the effects of the medium and the training on group processes. We expected trained CMC groups to establish more mutual understanding, to focus better on the judgmental task by showing more task

orientation and feedback, and to be more satisfied with the group process than untrained CMC groups (Hypothesis 3). In Table 3, the three forms of 
Table 3

\& Means, Standard Deviations, Contrast Estimates, and F Statistics of ANOVAs for Process and Outcome Variables in Computer-Mediated Groups Without and With Training and Face-to-Face Groups, With Chat Experience, Familiarity, and Gender Composition of the Group as Covariates

\begin{tabular}{|c|c|c|c|c|c|c|}
\hline Group Condition & Outcome Variable & $M(n=75)$ & $S D$ & Contrast Estimate $^{\mathrm{a}}$ & $F d f=2,70$ & $p$ \\
\hline & Coefficient of coherence & & & & & \\
\hline CMC no training & & 0.29 & 0.18 & & & \\
\hline CMC with training & & 0.54 & 0.14 & $0.24 * * *$ & & \\
\hline \multirow[t]{2}{*}{ Face to face } & & 0.68 & 0.10 & $0.38 * * *$ & 38.21 & .000 \\
\hline & Mutual understanding & & & & & \\
\hline CMC no training & & 4.57 & 0.64 & & & \\
\hline CMC with training & & 5.00 & 0.50 & $0.42 *$ & & \\
\hline \multirow[t]{2}{*}{ Face to face } & & 5.27 & 0.60 & $0.68^{* *}$ & 6.1 & .004 \\
\hline & Feedback & & & & & \\
\hline CMC no training & & 0.19 & 0.10 & & & \\
\hline CMC with training & & 0.29 & 0.10 & $0.10 * *$ & & \\
\hline \multirow[t]{2}{*}{ Face to face } & & 0.33 & 0.06 & $0.13^{* * *}$ & 8.74 & .000 \\
\hline & Task orientation & & & & & \\
\hline CMC no training & & 0.50 & 0.20 & & & \\
\hline CMC with training & & 0.68 & 0.18 & $0.17 * *$ & & \\
\hline \multirow[t]{2}{*}{ Face to face } & & 0.79 & 0.09 & $0.27 * * *$ & 14.68 & .000 \\
\hline & Satisfaction with group & sion & & & & \\
\hline CMC no training & & 3.64 & 1.14 & & & \\
\hline $\mathrm{CMC}$ with training & & 3.98 & 0.60 & 0.34 & & \\
\hline \multirow[t]{2}{*}{ Face to face } & & 4.73 & 0.63 & $1.6^{* * * *}$ & 12.93 & .000 \\
\hline & Interpersonal attraction & & & & & \\
\hline CMC no training & & 4.90 & 0.60 & & & \\
\hline CMC with training & & 5.00 & 0.57 & 0.09 & & \\
\hline Face to face & & 5.17 & 0.57 & 0.28 & 1.53 & 0.22 \\
\hline
\end{tabular}

Note. CMC = computer-mediated communication.

a. Contrast estimates were calculated by simple contrast analysis with the condition CMC without training as reference category. $* p<.05 . * * p<.01 . * * * p<.001$ 


\section{Cornelius, Boos • Enhancing Mutual Understanding}

training are presented as one combined training condition, because there were no significant differences in coherence between the different kinds of training.

Again, the relevant category for comparisons between conditions are CMC groups without training. A priori contrast analysis comparing FtF groups with CMC groups without training revealed that $\mathrm{FtF}$ groups perceived more mutual understanding than CMC groups without training. Trained CMC groups perceived more mutual understanding than untrained groups, too. Familiarity, chat experience, and gender composition had no effect on this result (all $F$ scores $<1.0$ ). Feedback to the statements of the other group members was higher in FtF groups and CMC groups with training than in CMC groups without training. Familiarity, chat experience, and gender composition did not mediate this effect. Note, however, that due to low interrater reliability of the feedback category, the likelihood of Type II error is given.

Although task orientation followed the same pattern as mutual understanding and feedback, familiarity played a role: Task orientation was lowest in identifiable groups, $F(1,70)=4.5, p<.05$.

Satisfaction with group process was higher in FtF than in untrained CMC groups. The difference between trained and untrained CMC groups was not significant. The covariates familiarity, chat experience, and gender composition did not affect this relation.

Interpersonal attraction was affected neither by media nor by training. Surprisingly, whether group members were unfamiliar or friends had no impact. No other covariate added further information.

Note that all effect sizes $\left(\eta^{2}\right)$ of familiarity were smaller than .10, whereas effect sizes of media and training comparison ranged from $\eta^{2}=.30$ for task orientation to $\eta^{2}=.53$ for coherence.

\section{Effects of Interpersonal Attraction}

on Satisfaction With Group Process

Contradicting Hypothesis 6, that $\mathrm{FtF}$ groups would reach consensus more often than CMC groups, in FtF $39.5 \%$ of groups reached consensus, whereas in CMC 59.5\% did. There was a slight tendency of CMC groups to reach consensus more often $\left(\chi^{2}=3.0, p=.07\right)$.

Did the lack of coherence even foster consensus in groups low in coherence? In the absence of a theoretical criterion, we used the median split to distinguish between groups high and low in coherence. Using the median of .70 as cutoff point, highly coherent $\mathrm{FtF}$ groups had more difficulties attaining consensus than $\mathrm{FtF}$ groups low in coherence $\left(\chi^{2}=4.2, p=.04\right)$. In CMC conditions, using the median split $(M d n=.52)$ as cutoff point, coherent and 
COMMUNICATION RESEARCH • April 2003

noncoherent groups did not differ in frequency of attaining consensus $\left(\chi^{2}=\right.$ $.04, p=.55)$. High coherence does not facilitate the attainment of consensus using either media.

Are highly coherent groups more satisfied with the decision process, even if they do not reach consensus? Hypotheses 4 and 5 proposed two alternative explanations for satisfaction with the group process: adequate coordination of opinions and proposals due to high coherence versus high interpersonal attraction and perceived mutual understanding due to commitment to the group. We ran a hierarchical regression analysis, entering gender composition, familiarity, chat experience, and training condition in the first step and examining the effects of the competing variables (coherence, interpersonal attraction, and mutual understanding) on satisfaction with the group process, dependent on media and attainment of consensus (see Table 4).

Coherence is the best predictor of satisfaction with group process in FtF groups without consensus. Interpersonal attraction predicts satisfaction with group process in FtF groups with consensus. Thus, FtF groups that reach consensus rely more on affiliation than on the actual communication process in reaching their agreement in judgments. Consensual FtF groups derive satisfaction from interpersonal attraction, nonconsensus FtF groups from coherence.

However, this pattern was not replicated in the other media condition. CMC groups without consensus were more satisfied the more mutual understanding they perceived. Mutual understanding is a positively evaluated group outcome that seems to compensate for the group's failure to reach consensus, especially in gender-heterogeneous groups. CMC groups with consensus were also most satisfied when mutual understanding was high, but this kind of perceived mutual understanding was not based on actual coherence. Coherence even reduced satisfaction with the group process in CMC groups that reached consensus.

\section{Conclusion}

Coherence and mutual understanding are reduced in synchronous CMC groups without training. Nondyadic exchange is the rule in this kind of CMC without media-adapted conversation management, leading to a situation in which typical patterns of FtF communication (including turn taking for the speakers and allocation of listener roles) are rescinded. Specific listener roles are no longer allocated, but messages are sent to an unspecified group addressee, which results in impaired feedback loops. Task orientation is lowest in unfamiliar, untrained CMC groups that reflect coordination and accommodation difficulties disturbing the grounding process. In untrained 
Table 4

Beta Coefficients and Results of Stepwise Regression Analyses for Alternative Explanations of

Satisfaction With Group Process in Consensus and Nonconsensus Groups

\begin{tabular}{|c|c|c|c|c|c|c|c|}
\hline Predictor & Condition & Variables in Equation & $\beta$ & $F$ & $d f$ & $p$ & Adjusted $R^{2}$ \\
\hline \multirow[t]{6}{*}{$\mathrm{CC}, \mathrm{MU}$, and interpersonal attraction } & FtF without consensus & $\mathrm{CC}$ & .60 & 12.3 & 1,21 & .002 & .34 \\
\hline & CMC without consensus & Homogeneity & -.56 & & & & \\
\hline & & MU & .45 & 5.8 & 2,12 & .02 & .40 \\
\hline & FtF with consensus & Interpersonal attraction & .76 & 17.6 & 1,13 & .001 & .54 \\
\hline & CMC with consensus & MU & .94 & 18.6 & 2,19 & .000 & .63 \\
\hline & & $\mathrm{CC}$ & -.40 & & & & \\
\hline
\end{tabular}

Note. $\mathrm{CC}=$ coefficient of coherence; $\mathrm{MU}=$ mutual understanding; $\mathrm{FtF}=$ face to face; $\mathrm{CMC}=$ computer-mediated communication. Gender composition (homogeneity vs. heterogeneity), familiarity, chat experience, and training were coded from dummy variables and entered in the first step of the regression analyses. 
COMMUNICATION RESEARCH • April 2003

CMC groups, the amount of disagreement and agreement during discussion does not meet the coordination requirements of judgmental tasks.

However, strategies for creating coherence and developing topics collaboratively in the computer medium can be trained, most effectively by reference strategies. Consequently, users can actively adapt their communication behavior to the media requirements and need not be passive victims of the technology. These results are not only relevant for work teams in organizational settings or students in virtual seminars but also for methodological reasons. Synchronous text-based CMC offers us the opportunity to study the effect of coherence and noncoherence on group processes. In CMC, coherence can be reliably manipulated by training. In $\mathrm{FtF}$, far too many context variables need to be controlled in order to achieve the same result.

One context variable could be identified in this study: familiarity. It fosters coherence, explicit references, and task orientation. Friends and other wellfamiliar participants orient their talk more toward their communication partners than toward only identifiable participants who may not expect prolonged interpersonal interaction. Maybe unfamiliar ad hoc groups need more time to establish routines and interpersonal relationship and thus cannot solely focus on the task. However, the effect of familiarity is rather small compared to the effect of training on coherence, explicit references, and task orientation.

Chat experience had no effect on media-adapted language use in CMC. One can only speculate about this nonexisting relationship. Besides the possibility that low interrater reliability led to this finding, the questionnaire asked in a rather unspecific manner if participants had ever chatted beforewe therefore do not know if respondents chatted as participants in taskoriented computer conferences or just for fun in public chat rooms. Chats are well known for excessive use of jokes, verbal games, and emoticons, but not for coherence (Herring, 1999). Thus, some experienced users might have been heavy chatters and resistant to the trade-off of coherent strategies.

In this study, coherence predicted mutual understanding but not interpersonal attraction. The perception of mutual understanding is not only based on actual coherence but also on interpersonal attraction, an aspect depending on factors others than those controlled for or measured in this study. Note that interpersonal attraction did not differ between identifiable and familiar groups or between groups of friends and was not influenced by training. This poses a challenge, because the notion of coherence implies that noncoherence should trigger fundamental attribution errors that lead to interpersonal misunderstandings (Bradac, 1988; Watzlawick, Beavin, \& Jackson, 1967). In German, the same wording (sich verstehen) signifies liking, understanding, and comprehension. Hence, an intercultural difference in language use may 


\section{Cornelius, Boos • Enhancing Mutual Understanding}

be the reason why the participants in this study did not perceive a link between coherence and affiliation as reported in the majority of American literature (Cappella, 1994; Tracy, 1985; Watzlawick et al., 1967). Given positive interpersonal attraction, high levels of mutual understanding will be perceived regardless of the actual level of coherence and the actual coordination of task communication in the group. A more simple explanation might be the lack of power of the coherence index because of insufficient interrater reliability. Still, this would not explain the existing relationship between coherence and mutual understanding.

Reduced coherence in CMC is accompanied by less feedback regarding decision proposals and opinions and by less task orientation, both of which make it difficult for the group to meet the task's coordination requirements. Consequently, CMC participants without training display a lower level of satisfaction with the decision process, although they actually reach consensus more often than do FtF groups.

Whereas coherence might facilitate converging in meaning, it seems to impair converging in attitudes toward consensus in a judgmental task. Contradicting media-fit theories and previous research, noncoherent CMC is the only condition that supports frequency of consensus. A possible explanation might be that deliberative and integrative communication processes based on coherence and mutual understanding prevent groups from coming to a false consensus. A false consensus neglects the divergent perspectives of the group members. This neglect does not have to be the result of social psychological processes such as groupthink or other influencing processes in groups but rather can simply be the consequence of a lack of understanding.

Whereas in $\mathrm{FtF}$ groups without consensus coherence predicts satisfaction with the group process, in CMC groups the perception of mutual understanding and satisfaction with the group process are not based on actual coherence but rather are effects of members feeling attracted to each other and perceiving themselves as a "good group." The feeling of being understood and of being heard is more central to eliciting convergence and consensus in a decision task than a comprehensive and deliberate discussion. Implicitly, this argument has been already made by Dennis and Valacich (1999), who labeled this stage of group process "affiliation" and not deliberation.

Several effects in the study pertain to cohesion as a result of having received training together with the other group members. Due to the fact that a direct measure of group salience is missing in this study, effects of variables such as interpersonal attraction and gender homogeneity in consensual groups were interpreted as cohesion. Recently, Yzerbyt, Castano, Leyens, and Paladino (2000) readdressed the concept of entitativity (Campbell, 1958), denoting the perception of the group as an entity versus an aggregate of 
COMMUNICATION RESEARCH • April 2003

single persons. They stress the psychological primacy of the in-group, which means that social cohesion and in-group identification in small groups are measurable in the absence of a relevant out-group. Sassenberg and Jonas (2000) developed an instrument for the measurement of group entitativity that still requires further validation but should be used in future studies to control for group salience.

In CMC groups that achieved consensus, coherence is negatively related to satisfaction with the group process, although it is nearly confounded with perceived mutual understanding. Coherence and comprehension may uncover divergent perspectives and attitudes that otherwise might be ignored in a chaotic conversational environment. It takes substantially more effort to integrate divergent perspectives into a group decision while simultaneously establishing coherence. Although coherent CMC groups might benefit from these efforts in mutual understanding, they might be overstrained by the demands of task instruction and of training instruction. Overstrain and frustration may lead to a negative evaluation of the group process.

Savicki, Kelley, and Ammon (2002) found similar effects of training on perceived satisfaction with the group process in CMC. They evaluated a training that should enhance the quality of the group processes in asynchronous, longterm mailing lists. In previous studies, a genderized style labeled as "high communication style," that is, self-disclosures, "I"-statements, and explicit references, had been observed and was positively correlated with perceived satisfaction with the group process (Savicki, Kelley, \& Oesterreich, 1998; Savicki, Lingenfelter, \& Kelley, 1996). This relationship could not be replicated when it did not occur spontaneously but had been-successfullymanipulated by training. Savicki et al. (2002) argued that trained communication patterns have to match naturally occurring communication patterns in order to enhance satisfaction with the group process. This may be the case in long-term, asynchronous mailing lists. However, in short-term, synchronous chat groups, time restriction and instructions (in our task coming to consensus and establishing coherence) impose a high strain on group members by which satisfaction may suffer. In future studies, training effectiveness and effects of trained communication patterns on task processes should be evaluated in separate experiments. Moreover, communication trainers and experimenters should not be identical (as was the case in the study at hand and in the study of Savicki et al., 2002).

In sum, the results demonstrate that reaching consensus is not the only appropriate criterion for evaluating the quality of group processes in shortterm decision-making groups with a given time frame. Process and outcome variables (coherence, task orientation, feedback, and mutual understanding) derived from coordination and communication theory are better suited to 


\section{Cornelius, Boos • Enhancing Mutual Understanding}

describe a group process in accordance with requirements of judgmental tasks. Still, mutual understanding based on conversational coherence does not foster the frequency of consensus as a relevant group outcome of decisionmaking groups. Conversely, coherence can be readily substituted by interpersonal attraction. Group members who like each other do not need to understand each other in order to reach consensus. Finally, in CMC the perception of the quality of the group process is influenced by the strain imposed by media accommodation, which might even be increased by the demands of training instructions.

This study reveals training effectiveness and effectiveness of mediaadapted conversation strategies on mutual understanding in a judgmental task. However, conversational coherence, mutual understanding, and convergence are multifaceted concepts constantly changing between deliberation and conformity and, in addition, interacting with group, task, and media features in a system of nested social contexts. Thus, this study has been just a first move toward disentangling these threads.

\section{Notes}

1. This research was supported by a grant from the Ministry of Education, Science and Culture of the Country of Lower Saxony, Germany, to the first author. We are indebted to Joseph E. McGrath for his substantial support and his revisions of an earlier version of this article. We would like to thank Kai J. Jonas and two anonymous reviewers for their critique and advice. Karl-Andrew Woltin helped us with the English language flow.

2. Groups with a dyad and a third person of different degrees of familiarity with the others were coded as follows: If the degrees of familiarity differed two levels from them, for example, identifiable and friends, the group familiarity was coded at the intermediate level, for example, familiar. If the degrees of familiarity differed only one level, for example, a dyad of friends and one person well known to them, the category "friends" was chosen to code the group familiarity.

\section{References}

Bakeman, R., \& Gottman, J. M. (1986). Observing interaction. An introduction to sequential analysis. Cambridge, UK: Cambridge University Press.

Bales, R. F. (1950). Interaction process analysis: A method for the study of small groups. Chicago: University of Chicago Press.

Boos, M., \& Sassenberg, K. (2001). Koordination in verteilten Arbeitsgruppen [Coordination in distributed work groups]. In E. H. Witte (Ed.), Leistungsverbesserungen in aufgabenorientierten Kleingruppen (pp. 198216). Lengerich, Germany: Pabst. 
COMMUNICATION RESEARCH • April 2003

Bradac, J. J. (1988). On coherence judgements and their multiple causes: A view from the message-variable paradigm. Commentary on Kellermann and Sleight. In J. A. Anderson (Ed.), Communication yearbook 12 (pp. 130145). Mahwah, NJ: Lawrence Erlbaum.

Brown, R. (2000). Group processes (2nd ed.). Oxford, UK: Blackwell.

Campbell, D. T. (1958). Common fate, similarity, and other indices of the status of aggregates of persons as social entities. Behavioral Science, 3, 14-25.

Cappella, J. N. (1994). The management of conversational interaction in adults and infants. In M. L. Knapp \& G. R. Miller (Eds.), Handbook of interpersonal communication (2nd ed.,pp. 380-418). Thousand Oaks, CA: Sage.

Clark, H. H., \& Brennan, S. (1993). Grounding in communication. In L. B. Resnick, J. M. Levine, \& S. D. Teasley (Eds.), Perspectives on socially shared cognition (pp. 127-149). Washington, DC: American Psychological Association.

Clark, H. H., \& Carlson, T. (1982). Hearers and speech acts. Language, 58, 332-373

Clark, H. H., \& Haviland, S. E. (1982). Comprehension and the given-new contract. In R. O. Freedle (Ed.), Discourse production and comprehension (2nd ed., pp. 1-41). Norwood, NJ: Ablex.

Clark, H. H., \& Wilkes-Gibbs, D. (1986). Referring as a collaborative process. Cognition, 22, 1-39.

Cohen, J. (1960). A coefficient of agreement for nominal scales. Educational and Psychological Measurement, 20, 37-46.

Condon, S. L., \& Cech, C. G. (1996). Functional comparison of face-to-face and computer-mediated decision making interactions. In S. C. Herring (Ed.), Computer-mediated communication: Linguistic, social and cross-cultural perspectives (pp. 65-80). Amsterdam: Benjamins.

Cramton, C. D. (2001). The mutual knowledge problem and its consequences for dispersed collaboration. Organizational Science, 12, 346-371.

Crow, B. K. (1983). Topic shifts in couples' conversation. In R. T. Craig. \& K. Tracy (Eds.), Conversational coherence (pp. 136-156). Beverly Hills, CA: Sage.

Dennis, A. R., \& Valacich, J. S. (1999). Rethinking media richness: Towards a theory of media synchronicity. In Proceedings of the $32^{\text {nd }}$ Hawaii International Conference on System Sciences, pp. 1-10.

Fleiss, J. L. (1981). Statistical methods for rates and proportions (2nd ed.). New York: Wiley.

Hellman, C. (1995). The notion of coherence in discourse. In G. Rickheith \& C. Habe (Eds.), Focus and coherence in discourse processing (pp. 190-202). Berlin, Germany: DeGruyter.

Herring, S. C. (1999). Interactional coherence in CMC. Journal of Computer Mediated Communication, 4(4). Retrieved June 6, 2001, from http://www. ascusc.org/jcmc/vol4/issue4/herring.html

Hogg, M. A. (1992). The social psychology of group cohesiveness: From attraction to social identity. London: Harvester Wheatsheaf.

Janis, I. L. (1972). Victims of groupthink. Boston: Houghton Mifflin. 


\section{Cornelius, Boos • Enhancing Mutual Understanding}

Kent, G. G., Davis, J. D., \& Shapiro, D. A. (1978). Resources required in the construction and reconstruction of conversation. Journal of Personality and Social Psychology, 36, 13-22.

Kiesler, S., \& Sproull, L. (1992). Group decision making and communication technology. Organizational Behavior and Human Decision Processes, 52, 96-123.

Kogan, N., \& Wallach, M. A. (1964). Risk taking: A study in cognition and personality. New York: Holt.

Lott, A. J., \& Lott, B. E. (1965). Group cohesiveness as interpersonal attraction. Psychological Bulletin, 64, 259-309.

McGrath, J. E. (1984). Groups: Interaction and performance. Englewood Cliffs, NJ: Prentice Hall.

McGrath, J. E. (1990). Time matters in groups. In J. Galegher, R. E. Kraut, \& C. Egido, (Eds.), Intellectual teamwork: Social and technological foundations of cooperative work (pp. 111-145). Hillsdale, NJ: Lawrence Erlbaum.

McGrath, J. E., \& Hollingshead, A. B. (1994). Groups interacting with technology. Newbury Park, CA: Sage.

Ng, S. H., \& Bradac, J. J. (1993). Power in language: Verbal communication and social influence. Newbury Park, CA: Sage.

Oehlschlegel, S., \& Piontkowski, U. (1997). Topic progression and social categorization. Journal of Language and Social Psychology, 16, 444-455.

Parker, K. C. H. (1988). Speaking turns in small group interaction: A contextsensitive event sequence model. Journal of Personality and Social Psychology, 54, 965-971.

Planalp, S., \& Tracy, K. (1980). Not to change the topic but: A cognitive approach to the management of conversations. In D. D. Nimmo (Ed.), Communication yearbook 4 (pp. 237-258). New Brunswick, NJ: Transaction Books.

Reichman, R. (1978). Conversational coherency. Cognitive Science, 2, 283327.

Reid, S. A., \& Ng, S. H. (2000). Conversation as a resource for influence: Evidence for prototypical arguments and social identification processes. European Journal of Social Psychology, 30, 83-100.

Reimer, T., Neuser, A., \& Schmitt, C. (1997). Unter welchen Bedingungen erhöht die Kommunikation zwischen den Gruppenmitgliedern die Koordinationsleistung in einer Kleingruppe? [Under which circumstances does communication between group members increase the performance of a small group?]. Zeitschrift für experimentelle Psychologie, 44, 495-518.

Sassenberg, K., \& Jonas, K. J. (2000, March). Entitativity as basis for the selfcategorization as member of a (small) group. Paper presented at the $42 \mathrm{nd}$ Meeting of Experimental Psychologists, Braunschweig, Germany.

Sassenberg, K., \& Postmes, T. (2000). Cognitive and strategic processes in small groups: Effects of anonymity of the self and anonymity of the group on social influence. Unpublished working paper, Friedrich-SchillerUniversity Jena/University of Amsterdam. 
COMMUNICATION RESEARCH • April 2003

Savicki, V., Kelley, M., \& Ammon, B. (2002). Effects of training on computermediated communication in single or mixed gender small task groups. Computers in Human Behavior, 18, 257-269.

Savicki, V., Kelley, M., \& Oesterreich, E. (1998). Effects of instructions on computer-mediated communication in single- oder mixed-gender small task groups. Computers in Human Behavior, 14, 163-180.

Savicki, V., Lingenfelter, D., \& Kelley, M. (1996). Gender language style and group composition in Internet discussion groups. Journal of Computer Mediated Communication, 2(3). Retrieved June 6, 2001, from http://www. ascusc.org/jmc/vol2/issue3/savicki.htm

Schegloff, E. A. (1990). On the organization of sequences as a source of coherence in talk-in-interaction. In B. Dorval (Ed.), Conversational organization and its development (pp. 51-77). Norwood, NJ: Ablex.

Schwarz, N. (2000). Social judgments and attitudes: Warmer, more social, and less conscious. European Journal of Social Psychology, 30, 149-176.

Spears, R., \& Lea, M. (1992). Social influence and the influence of the "social" in computer-mediated communication. In M. Lea (Ed.), Contexts of computer-mediated communication (pp. 30-65). Harvester, UK: Wheatsheaf.

Sperber, D., \& Wilson, D. (1986). Relevance: Communication and cognition. Cambridge, MA: Harvard University Press.

Stasser, G., \& Taylor, L. A. (1991). Speaking turns in face-to-face discussions. Journal of Personality and Social Psychology, 60, 675-684.

Straus, S. G. (1996). Getting a clue: The effects of communication media and information distribution on participation and performance in computermediated and face-to-face groups. Small Group Research, 27, 115-142.

Straus, S. G. (1999). Testing a typology of tasks: An empirical validation of McGrath (1984) group task circumplex model. Small Group Research, 30, 166-187.

Straus, S. G., \& McGrath, J. E. (1994). Does the medium matter? The interaction of task type and technology on group performance and member reactions. Journal of Applied Psychology, 79, 87-97.

Tracy, K. (1985). Conversational coherence: A cognitively grounded rules approach. In R. L. Street \& J. N. Cappella (Eds.), Sequence and pattern in communicative behaviour (pp. 30-49). London: Edward Arnold.

Tschan, F. (1995). Communication enhances small group performance if it conforms to task requirements: The concept of ideal communication cycles. Basic and Applied Social Psychology, 17, 371-393.

Turner, J. C. (1991). Group polarization. In J. C. Turner (Ed.), Social influence (pp. 49-79). Pacific Grove, CA: Brooks/Cole.

Walther, J. B. (1994). Anticipated ongoing interaction versus channel effects on relational communication in computer-mediated interaction. Human Communication Research, 20, 473-501.

Walther, J. B. (1996). Computer-mediated communication: Impersonal, interpersonal and hyperpersonal interaction. Human Communication Research, 23, 1-43. 


\section{Cornelius, Boos • Enhancing Mutual Understanding}

Walther, J. B., Anderson, J. F., \& Park, D. W. (1994). Interpersonal effects in computer-mediated interaction. Human Communication Research, 23, 143.

Walther, J. B., Boos, M., \& Jonas, K. J. (2002). Misattribution and attributional redirection in distributed virtual groups. IEEE Proceedings of the Hawai'i International Conference on System Sciences, pp. 7-10.

Watzlawick, P., Beavin, J., \& Jackson, D. D. (1967). Pragmatics of human communication. New York: Norton.

Werner, C., \& Latané, B. (1976). Responsiveness and communication medium in dyadic interactions. Bulletin of Psychonomic Society, 8, 13-15.

Yzerbyt, V., Castano, E., Leyens, J.-P., \& Paladino, M.-P. (2000). The primacy of the ingroup: The interplay of entitativity and identification. European Review of Social Psychology, 11, 257-295.

Zajonc, R. B. (1960). The process of cognitive tuning and communication. Journal of Abnormal Social Psychology, 61, 159-167.

Caroline Cornelius holds a Ph.D. in psychology from the University of Göttingen. She is working as an e-moderator, online trainer, and teletutor.

Margarete Boos is professor of Social and Communication Psychology at the Georg-Elias-Müller-Institute of Psychology, University of Göttingen, Germany. Her main research areas are decision making and computer-mediated communication in groups and organizations. 